\title{
Diacronie
}

Studi di Storia Contemporanea

$N^{\circ} 24,4 \mid 2015$

Le dittature militari: fisionomia ed eredità politica

\section{Franco Andreucci, Da Gramsci a Occhetto. Nobiltà e miseria del Pci 1921-1991}

\section{Michelangela Di Giacomo}

\section{Q OpenEdition}

Edizione digitale

URL: http://journals.openedition.org/diacronie/3606

DOI: $10.4000 /$ diacronie.3606

ISSN: 2038-0925

\section{Editore}

Association culturelle Diacronie

\section{Notizia bibliografica digitale}

Michelangela Di Giacomo, « Franco Andreucci, Da Gramsci a Occhetto. Nobiltà e miseria del Pci 1921-1997 », Diacronie [Online], № 24, 4 | 2015, documento 23, Messo online il 29 décembre 2015, consultato il 24 septembre 2020. URL : http://journals.openedition.org/diacronie/3606 ; DOI : https:// doi.org/10.4000/diacronie.3606 


\section{Diacronie}

N. 24 | 4|2015 Le dittature militari: fisionomia ed eredità politica

23/

\section{RECENSIONE:}

\section{Franco ANDREUCCI, Da Gramsci a Occhetto. Nobiltà e miseria del Pci 1921-1991, Pisa, Della Porta editori, 2014, 467 pp.}

a cura di Michelangela DI GIACOMO *

Franco Andreucci, professore di storia contemporanea presso l'Università di Pisa sino al 2013, già attivista del Pci, è indubbiamente noto per la sua edizione, risalente al 1979, delle Opere di Togliatti per i tipi degli Editori Riuniti, la casa editrice di quel partito $^{1}$. Tuttavia, e paradossalmente, è forse più noto per la sua ascesa alla ribalta delle cronache con la pubblicazione, nell'ormai lontano 1992, di una lettera inedita di Togliatti da lui ritrovata negli archivi del Comintern, la cui traduzione ed esegesi fu oggetto di importanti e pubblici dibattiti che vennero a inquinare la già difficile campagna elettorale di quell'anno ${ }^{2}$. La vicenda fu più volte riaperta nei decenni successivi, più che nel consesso accademico - riguardo alla quale pure sono intervenuti nomi di calibro da Giuseppe Vacca a Giovanni Sabbatucci - in quello mediatico: basti vedere le recensioni al precedente volume di Andreucci Falce e martello. Identità e linguaggi dei comunisti italiani fra stalinismo e guerra fredda ${ }^{3}$, che rimandano tutte alla dimensione "pubblica" della storia. In quel volume - sosteneva Aldo Agosti recensendolo per l'Indice dei libri del mese - l'obiettivo

\footnotetext{
${ }^{1}$ TOGLIATTI, Palmiro [SPRIANO, Paolo, ANDREUCCI, Franco (a cura di)], Opere, Vol. IV, 1935-1944, , Roma, Editori Riuniti, 1979.

2 Per la vicenda basta vedere le cronache dell'epoca, URL:

< http://ricerca.repubblica.it/repubblica/archivio/repubblica/1992/02/14/manipolata-lalettera-di-togliatti.html >;

$<\quad$ http://ricerca.repubblica.it/repubblica/archivio/repubblica/1992/o2/15/la-waterloo-diandreucci.html >;

$<$ http://ricerca.repubblica.it/repubblica/archivio/repubblica/1992/03/27/andreucci-finiscesott-inchiesta-per-il-falso.html > [consultati il 25 maggio 2015].

3 ANDREUCCI, Franco, Falce e Martello. Identità e linguaggi dei comunisti italiani fra stalinismo e guerra fredda, Bologna, Bononia University Press, 2005.
} 
è - dichiaratamente - la critica di un complicato intreccio di tradizione di partito, memoria e storiografia che prende forma nel Pci fra gli anni trenta e l'inizio degli anni cinquanta, e che, a giudizio di Andreucci, condiziona profondamente l'identità del partito, lasciando sopravvivere intatto nel tempo un nocciolo duro di stalinismo, fatto di strutture logiche dogmatiche, di un linguaggio esoterico, di un complesso di riti e simboli che configurano una subcultura chiusa in sé stessa.

Per dimostrare questa tesi l'autore fornisce un ampio e impietoso repertorio di citazioni dalla stampa comunista degli anni compresi fra il 1947 e il 1956, che costituisce un vero campionario di propaganda stalinista rozza e manichea. Partendo di qui, Andreucci sostiene non solo che «le idee dell'autonomia e della diversità non possano essere retrodatate» (il che, per la verità, sfonda una porta aperta già da molti anni da quella "tradizione storica" che è il suo idolo polemico), ma anche che «dovrebbe essere sottoposta a profonda revisione l'idea che il Pci abbia contribuito all'educazione politica delle classi subalterne», e che bisognerebbe mettere l'accento non sul suo apporto alla formazione di "moderni cittadini”, ma su «una preparazione ideologica di massa, fondata su radicalizzazione estreme» 4 .

A conclusione di quella nota, Agosti, che si sentiva tra l'altro chiamato in causa nel tentativo di fare di tutta l'erba un fascio con la storiografia sul Pci - additandola sostanzialmente come una storiografia del Pci anche laddove le appartenenze politiche non erano di quel partito - segnalava ad Andreucci l'opportunità di spingersi oltre il 1956 nella sua analisi. Il volume Da Gramsci a Occhetto è in qualche misura la trascrizione in pratica di quel consiglio, ma al tempo stesso una trascrizione alquanto squilibrata: con ciò non intendiamo tanto sul piano teorico, quanto, piuttosto, sul piano spaziale. Lo spazio dedicato alle vicende post-1956 è, infatti, minimo in confronto all'ampia trattazione di quelle precedenti a quella cesura, quasi una corsa in discesa che conchiude in due capitoli su otto le trasformazioni del partito da allora sino al 1989 condensando addirittura le Segreterie di Berlinguer, Natta e Occhetto, nel residuale spazio di un capitolo intitolato La caduta del comunismo e la fine del Pci. Il tema, comunque, rimane lo stesso del volume precedente: spiegare quanto il Pci fosse permeato di stalinismo a tutti i suoi livelli - si salvano i dirigenti locali del crinale degli anni Cinquanta e quelli delle prime "giunte rosse" - e quanto inventata sia stata la tradizione del partito come entità intrinsecamente italiana. La diversità del Pci fu

\footnotetext{
4 AGOSTI, Aldo, «Una storiografia sotto accusa», in L’indice dei libri del mese, 22, 12, 2005, p. 20, disponibile anche al link, URL: http://www.digibess.it/fedora/repository/lindicescarl:LINDICE-00247-0020 > [consultato il 25 maggio 2015].
} 
dunque tutta insita nel suo proclamarsi tale? Per rispondere a tale domanda - e a molte altre che riguardano, da un lato, l'inconsueto radicamento di un partito comunista in ampie fasce sociali e geografiche della penisola e, dall'altro, l'altrettanto inconsueta repentina conclusione della sua parabola - l'autore si concentra sui dirigenti del partito, rivalutando al contempo autori "altri” quali Guareschi, Silone e Gorresio e lasciando ampio respiro a riflessioni sul panorama linguistico, visivo e musicale del partito - come già nel caso del suo volume precedentes, tra le parti più coinvolgenti e originali del libro.

Nel dover esprimere un giudizio equilibrato su tale lettura, dunque, non si può non sentirsi chiamati in causa nelle proprie convinzioni personali riguardo alla vicenda del Pci nell'Italia repubblicana. In altri termini: ha ragione Andreucci e molte delle nostre certezze sul Pci sono di fatto distorte dal "prima" della tradizione inventata o ha torto Andreucci nel suo a volte impietoso ritratto di un Pci permeato di grigiore burocratico? E nel dargli torto, non staremmo usando gli strumenti che ci ha lasciato quella stessa tradizione che egli accusa? Evidentemente la domanda esula dal render conto del volume, ma il dubbio stesso segnala la rilevanza del tema scelto: a trent'anni di distanza dalla conclusione della vicenda comunista in Italia - indipendentemente da quale sia il valore che ai suoi "eredi” o "epigoni” si voglia attribuire - essa è ancora una scintilla di appassionate riflessioni, un'eredità molto più pesante di quanto l'autore non sembri invece ritenere. In tal senso riteniamo che il libro di Andreucci sia comunque da leggere, indipendentemente da quanto ci si possa trovare d'accordo con i suoi giudizi: si configura in qualche modo come una storia d'Italia e del mondo vista attraverso il prisma del Pci, ripercorrendo tutte le grandi tappe della storia del Novecento con gli occhi di un soggetto che nel bene e nel male ha caratterizzato il secolo appena trascorso. Che se ne scriva in parte per accusarlo non toglie minimamente forza al riconoscimento del suo ruolo storico peculiare nelle vicende novecentesche.

Prima di entrare nel dettaglio del libro, ci sembra di dover chiarire il punto in cui esso debba essere collocato nell'ambito degli studi a tema storico. Si tratta - a nostro parere, ma anche nelle parole dell'autore che lo definisce una «fascinating narrative ${ }^{6}$ commissionatagli dall'editore per «rendere conto dell'esistenza di una copiosa letteratura sulla storia del Pci» ${ }^{7}$ - di un esperimento alquanto ben riuscito di "Public History", tanto nell'accezione peggiorativa di distorsione, quanto in quella neutra di divulgazione. Anzitutto occorre segnalare che in quasi cinquecento pagine di volume

5 ANDREUCCI, Franco, Da Gramsci a Occhetto. Nobiltà e miseria del Pci 1921-1991, Pisa, Della Porta editori, 2014.

${ }^{6}$ ANDREUCCI, Franco, op. cit., p. 29.

7 Ibidem. 
non vi è neanche una nota - che, per quanto sopravvalutate nonché noiose, sono emblema della riconosciuta scientificità di un saggio storiografico. In secondo luogo, vi è da dire che la pur ampia bibliografia non esce minimamente dal tracciato dell'ordinario: si rende conto dei grandi nomi della letteratura sul Pci - in massima parte italiani - ma è scarsamente rappresentato tanto il dibattito sulle riviste quanto la ormai vasta produzione sui temi del Pci di "giovani storici" e si notano anche alcune grandi assenze (ci viene in mente la biografia di Natta a cura di Paolo Turi, ad esempio $^{8}$, sebbene sia un'assenza giustificata dal ruolo del tutto marginale che è attribuito al segretario nelle vicende finali del partito: vi trova infatti quasi più spazio Cossutta di Natta). In terzo luogo, conviene far notare come il sottotitolo richiami alla mente - ne è consapevole l'autore? - un'operazione simile realizzata in Spagna alcuni anni orsono col volume Miseria y grandeza del Pce di Gregorio Morán', anch'egli ex comunista passato a posizioni critiche - e, come in quel caso, la domanda è: qual è la grandezza e quale la miseria? Ad un primo impatto, infatti, sembrerebbe scontato rispondere che la grandezza sia stata quella dell'espansione e del radicamento e la miseria quella dell'agonia finale, ma ad una più approfondita lettura i due termini sembrano forse da invertire.

Chiarito dunque il tipo di libro cui il lettore va incontro, possiamo senza tema affermare che in tal senso il lavoro sia assolutamente ben fatto. Anzitutto perché si legge con una certa inusuale scorrevolezza - ricorda nella prosa più Wu Ming che un manuale - e soprattutto perché appassiona, spiega le vicende umane dei singoli personaggi che appaiono sulla scena quasi come fossero i protagonisti di un romanzo. Il che, in tempi di scarsi lettori di studi storici accademici e di numerosi appassionati di storia-giornalismo, è evidentemente un pregio del volume. In secondo luogo, il libro mantiene in qualche modo una visione "indefinita" dell'oggetto di studio, con una certa dose di onestà intellettuale. Si presenta un Pci dai molteplici volti: non è fatto segreto alcuno dell'“antipatia” nutrita nei confronti di Togliatti e della speculare simpatia per un Gramsci che è vittima prima dei suoi compagni che del fascismo, ma al tempo stesso si racconta un Pci eroico fatto di buoni con grandi propositi, sognatori, anarchici, volenterosi - tutti con tristi destini, storie tragiche e appassionate che possono senza dubbio affascinare il lettore al pari di un romanzo di avventura. Il Pci è dunque un partito di sconfitti nella sconfitta. Al Pci si riconosce «una straordinaria disponibilità ed un'eccellente capacità di governare e dirigere i conflitti sociali e in particolare quelli -

8 TURI, Paolo, L'ultimo Segretario. Vita e carriera di Alessandro Natta, Bologna, Cedam, 1996.

9 MORÁN, Gregorio, Miseria y grandeza del PartidoComunista de España 1939-1985, Barcelona, Planeta, 1986. 
tipici degli anni fra i Cinquanta e i Settanta - che partono dalle classi inferiori» ${ }^{10}$, senza che questo lo renda un partito meno "stalinista", settario, grigio e disciplinato. Ora, paradossalmente, proprio quella disciplina e quella capacità di organizzazione risultano dal volume come i punti di forza del partito che, andandosi a sommare alla capacità di «acquisire le eredità del passato e di mantenerle al di là dei cambiamenti più profondi nella società» ${ }^{11}$, gli hanno consentito di diventare un partito di massa moderno ed efficiente, in grado di rappresentare un ampio spettro di interessi sociali e di attrarre gli intellettuali. Nonostante ciò, nell'ottica dell'autore il Pci è privato di qualsivoglia capacità programmatica reale, è visto più come il partito delle grandi frasi ad effetto che della governabilità, tranne forse un ammorbidimento del giudizio in alcuni punti, nei momenti in cui si descrive il Pci come più socialdemocratico di quanto i suoi dirigenti Berlinguer in primis - non intendessero ammettere. La questione è: sono questi valori o disvalori? E parimenti: se si riconosce che il Pci si è estinto quando non è più stato in grado di riprodurre e divulgare la propria tradizione - identità storica e cultura politica - è un valore o un disvalore il fatto che esso l'abbia negli anni codificata tanto da farne base della propria legittimità e presupposto della propria permanenza in vita?

I fattori storia/continuità/tradizione/innovazione/narrazione percorrono tutto il lavoro di Andreucci che non a caso si apre con il 1914, ben prima, dunque, della nascita ufficiale dell'oggetto di studio. Lo stesso panorama linguistico ed ideale del partito è sempre sottoposto ad un controllo che ne ricerca analogie o differenze con quelli del movimento operaio italiano ed internazionale. Così come un filo rosso è il rapporto tra partito e intellettuali - storici, letterati, accademici - segnalando come vi fosse una evidente dicotomia tra il volersi raffigurare partito degli operai ed essere invece il catalizzatore delle simpatie di studenti, architetti, registi e pensatori di ogni genere. Tra l'altro, proprio perché tale attrazione era indipendente dalla volontà dei dirigenti del Pci, la linea culturale ufficiale del partito sarebbe stata sempre gretta e oscurantista. L'autore riconosce a Togliatti le sue altissime virtù intellettuali, la qualità dei prodotti del suo pensiero, il fatto stesso che l'“operazione Gramsci” non era «né scontata né obbligata ${ }^{12}$, ma al tempo stesso gli attribuisce uno stalinismo fiero e quasi sino all'ultimo inscalfibile. Fortissimi e continui sono poi i rimandi alla collocazione internazionale del Pci, con giudizi severi sull'incapacità o impossibilità o mancata volontà di emendarsi dal patronage sovietico - e sui riflessi dogmatici che tale tutela aveva nell'impostazione educativa del Pci in Italia. Al Pci si riconosce dunque valore coesivo, come cemento delle appartenenze, ma gli si nega quello educativo. Così come

${ }^{10}$ ANDREUCCI, Franco, Da Gramsci a Occhetto, cit., p. 19.

${ }^{11}$ Ibidem, p. 21.

${ }^{12}$ Ibidem, p. 277. 
al contempo gli si nega la funzione nazionalizzatrice dei lavoratori attribuendogli invece una capacità distorsiva di quel processo di integrazione nazionale - con la formazione di una subcultura chiusa che stimolava l'appartenenza non allo Stato ma ad un'area identitaria diversa ed altra. Stonano forse un po' dunque i risultati elettorali del partito: possono spiegarsi con la capacità di fornire un discorso semplicistico e manicheo ma enfatico e coinvolgente tutto imperniato sulla vertente bene $v s$. male? Una soluzione talmente poco convincente che lo stesso autore deve ammettere che le proposte politiche del Pci «si arricchiscono e ricevono adesioni anche fuori dalla loro tradizionale influenza. Accanto ai temi consueti della lotta ai monopoli e della riforma agraria, si formulano obiettivi nuovi nella destinazione della spesa pubblica: la casa [...], i trasporti; il diritto allo studio» ${ }^{13}$.

Il libro si articola lungo nove capitoli, più una relativamente amplia bibliografia e una introduzione metodologica. La struttura è equilibrata in termini di dimensioni - a tutti i capitoli è dato uno spazio analogo, con l'eccezione del primo (sulla formazione del Pci) e dell'ultimo (sulla caduta del comunismo e la fine del Pci). Il primo copre dal 1914 - con la preparazione del Congresso sulla Pace che l'Internazionale socialista aveva deciso di tenere a Vienna - alla fondazione del Pci, attraversa la Grande Guerra, segue il passaggio della nuova generazione dal socialismo al comunismo; il secondo copre gli anni Venti, seguendo il passaggio alla clandestinità o all'esilio, l'incarcerazione di Gramsci, tracciando i profili dei principali protagonisti, da Longo a Secchia a Togliatti. Il terzo capitolo è dedicato al Pci sotto le dittature e al rafforzamento del legame con l'Unione Sovietica attraverso la presenza degli italiani a Mosca e la Guerra di Spagna. Il quarto si occupa dell'Antifascismo e della Resistenza, comprendendovi la "Svolta di Salerno" e la costruzione delle future alleanze. Il quinto copre gli anni Cinquanta - ricostruzione, stalinismo e guerra fredda con le scelte legate alla collocazione internazionale del Paese e al radicamento delle doppie lealtà e della conventio ad excludendum nei confronti del Pci. Qui vasto spazio è dedicato alla storia culturale e degli intellettuali, mentre il ragionamento identitario sull'essere comunisti occupa l'intero capitolo sesto. Gli fa seguito un capitolo dedicato alla prima crisi interna al partito, avvenuta con la morte di Stalin e il 1956. Gli anni Sessanta sono considerati nel capitolo ottavo come una "guerra di posizione", al cui termine si intravede una nuova Italia "di sinistra" sorta nel '68. Nell'ultimo capitolo, quello della caduta del comunismo, confluiscono anche Berlinguer e l'eurocomunismo, che si chiude con il 1991, all'apertura del Congresso di Rimini.

13 Ibidem, p. 377. 
Il Pci di Andreucci, dunque, è un po' schizofrenico: capace di esserci nei momenti chiave della trasformazione nazionale, incapace di dare letture al passo coi tempi (dal Fascismo al miracolo economico al compromesso storico, sembrano essere, quelle del Pci, tutte cattive interpretazioni della situazione del Paese - e del mondo); chiuso e settario, ma capace di coinvolgere grandi masse. Insomma, a tutti gli effetti, un partito a cui si riconosce una grandezza - d'altronde alquanto innegabile - ma di cui si enfatizza la miseria - anch'essa d'altro canto sufficientemente messa in evidenza dagli studi pubblicati negli ultimi, almeno, vent'anni. 


\section{* L'autore}

Michelangela Di Giacomo si è addottorata presso l'Università di Siena; è Borsista presso l'Institut d'Estudis Catalans di Barcellona, è Cultore della materia presso il Dispi-Università di Siena. Ha vinto con le sue tesi il premio della Presidenza della Repubblica/Fondazione Spadolini nel 2012 e di quello del Senato della Repubblica nel 2009. È autrice del volume Da Porta Nuova a Corso Traiano. Movimento operaio e immigrazione meridionale a Torino. 1955-1969 (Bologna, BUP, 2013). Ha pubblicato saggi sulle riviste «Storiografia» (2009), «Studi Storici» (2010), «Memoria e Ricerca» (2012), «Historia, Trabajo y Sociedad» (2013).

URL: < http://www.studistorici.com/progett/autori/\#DiGiacomo >

\section{Per citare questo articolo:}

DI GIACOMO, Michelangela, «Recensione: Franco ANDREUCCI, Da Gramsci a Occhetto. Nobiltà e miseria del Pci 1921-1991, Pisa, Della Porta editori, 2014, 467 pp.», Diacronie. Studi di Storia Contemporanea : Le dittature militari: fisionomia ed eredità politica, 29/12/2015,

URL:<http://www.studistorici.com/2015/12/29/digiacomo_numero_24/ >

Diacronie Studi di Storia Contemporanea $\beta$ www.diacronie.it

Risorsa digitale indipendente a carattere storiografico. Uscita trimestrale. redazione.diacronie@hotmail.it

Comitato di redazione: Jacopo Bassi - Luca Bufarale - Elisa Grandi - Antonio César Moreno Cantano - Deborah Paci - Fausto Pietrancosta - Alessandro Salvador - Matteo Tomasoni - Luca Zuccolo

Diritti: gli articoli di Diacronie. Studi di Storia Contemporanea sono pubblicati sotto licenza Creative Commons 3.0. Possono essere riprodotti e modificati a patto di indicare eventuali modifiche dei contenuti, di riconoscere la paternità dell'opera e di condividerla allo stesso modo. La citazione di estratti è comunque sempre autorizzata, nei limiti previsti dalla legge. 